\title{
A gated single-photon avalanche diode array fabricated in a conventional CMOS process for triggered systems
}

\author{
E. Vilella*, A. Diéguez \\ Department of Electronics, University of Barcelona (UB), C/Martí i Franquès 1, 08028, Barcelona Spain
}

\section{A R T I C L E I N F O}

\section{Article history:}

Received 29 September 2011

Received in revised form 2 December 2011

Accepted 10 January 2012

Available online xxx

\section{Keywords}

Single-photon avalanche diode (SPAD)

Afterpulsing

CMOS

Dark count

Gated operation

Low noise

\begin{abstract}
A B S T R A C T
A bidimensional array based on single-photon avalanche diodes for triggered imaging systems is presented. The diodes are operated in the gated mode of acquisition to reduce the probability to detect noise counts interfering with photon arrival events. In addition, low reverse bias overvoltages are used to lessen the dark count rate. Experimental results demonstrate that the prototype fabricated with a standard HV-CMOS process gets rid of afterpulses and offers a reduced dark count probability by applying the proposed modes of operation. The detector exhibits a dynamic range of 15 bits with short gated 'on' periods of $10 \mathrm{~ns}$ and a reverse bias overvoltage of $1.0 \mathrm{~V}$.
\end{abstract}

(c) 2012 Elsevier B.V. All rights reserved.

\section{Introduction}

Solid-state devices have been used for the detection of radiation, mainly light, since the 1960s. At present time, there exists a good number of sensor technologies and more or less well established. Typical sensors available in the market are charge coupled devices (CCDs) [1], monolithic active pixel sensors (MAPS) [2] and silicon photomultipliers (SiPMs) [3]. CCDs are imaging sensors, whereas SiPMs cannot provide any spatial resolution. MAPS are used for the detection of ionizing particles. Although these sensors can be easily found in cameras, videos and also in the bio-medical field, they cannot supply the required readout speed or temporal and spatial resolution required by many other potential applications. Such a gap in the market has stimulated research and development to improve the already mature technologies but also to explore new ones, like for instance column parallel CCDs (CPCCDs) [4] and depleted field effect transistors (DEPFETs) [5]. DEPFETs offer twodimensional resolution and are being mainly developed for the detection of ionizing particles. In addition, single-photon avalanche diodes (SPADs) (or alternatively Geiger-mode APDs or GAPDs), which provide an extremely high sensitivity and excellent time resolution that allow to detect single photons in picosecond rise times [6], have regained interest due to the recent achievement to monolithically integrate the sensor and the readout electronics on a

\footnotetext{
* Corresponding author. Tel.: +34 934039 157; fax: +34 934021148.

E-mail address: evilella@el.ub.es (E. Vilella).
}

single CMOS die [7]. However, these sensors suffer from high levels of intrinsic noise that degrade their performance.

Each technology has its own advantages and drawbacks, so the appropriate one must be chosen according to the requirements of the application. In this article, we present an SPAD detector with the sensor and the front-end electronics monolithically integrated with the standard HV-AMS $0.35 \mu \mathrm{m}$ CMOS technology (h35b4). Two main techniques have been implemented to reduce the noise. On the one hand, the sensor can be operated in a gated mode to reduce the probability to detect the noise. On the other hand, the sensor can function with low biases to reduce the intrinsic noise while the photon detection efficiency is still good enough. No cooling methods or digital processing have been used to diminish noise figures. The detector was especially designed regarding applications related with high energy physics experiments. In particular, it was designed to serve as a tracker detector to reconstruct the particle path in future linear colliders [8]. However, it could be used in any other application that operates with a trigger signal, such as three-dimensional imaging, fluorescence spectroscopy and time-of-flight ranging.

\section{Single-photon avalanche diodes}

An SPAD is based on a $\mathrm{p}-\mathrm{n}$ junction reverse biased above its breakdown voltage $\left(V_{\mathrm{BD}}\right)$ in the so-called Geiger mode. At this polarization, there exists a very high electric field within the junction area forming the avalanche region. If a free carrier having more energy than the band gap of the material reaches the 
avalanche region, it can generate an $\mathrm{e}^{-}-\mathrm{h}^{+}$pair. This $\mathrm{e}^{-}-\mathrm{h}^{+}$pair can be accelerated by the high electric field up to the point at which it can generate another $\mathrm{e}^{-}-\mathrm{h}^{+}$pair by impact ionization. The new pair can be accelerated as well, thus starting an avalanche multiplication process that gives rise to the prompt generation of a detectable macroscopic current pulse. This process results in an internal gain of between $10^{5}$ and $10^{6}$. However, since the avalanche is self-sustained, the current continues to flow and it needs to be stopped in order to avoid burning the device. This operation is performed by the quenching electronics, typically implemented by means of a resistor placed in series with the sensor, by lowering the reverse bias voltage down to or below $V_{\mathrm{BD}}$. Finally, the bias of the sensor has to be restored so that the sensor is made sensitive again for upcoming avalanches.

Despite the extraordinary efficiency of SPADs in single photon detection, there are however some drawbacks and limitations. Thermal and tunneling generated carriers within the $\mathrm{p}-\mathrm{n}$ junction can also trigger avalanche pulses that are indistinguishable from actual radiation-triggered pulses. The frequency of generation of these spurious pulses, known as dark count rate (DCR, usually expressed in $\mathrm{Hz}$ ), depends on the doping profile, the quality of the technology, the reverse bias overvoltage $\left(V_{\mathrm{OV}}\right)$ over $V_{\mathrm{BD}}$ and the temperature. Moreover, charge carriers that were trapped by crystal defects during an avalanche flow also generate false pulses when they are released at a certain time delay and re-trigger the SPAD. The afterpulsing probability is a function of the trap density, the number of carriers generated during an avalanche and the release time of these carriers. Both dark counts and afterpulses degrade the performance of the sensor, leading to erroneous results in yes/no applications and limiting the range of detectable signals in light intensity measurements. In addition, they also increase the amount of data that has to be stored for the subsequent processing in which signal is discriminated from noise. Apart from that,

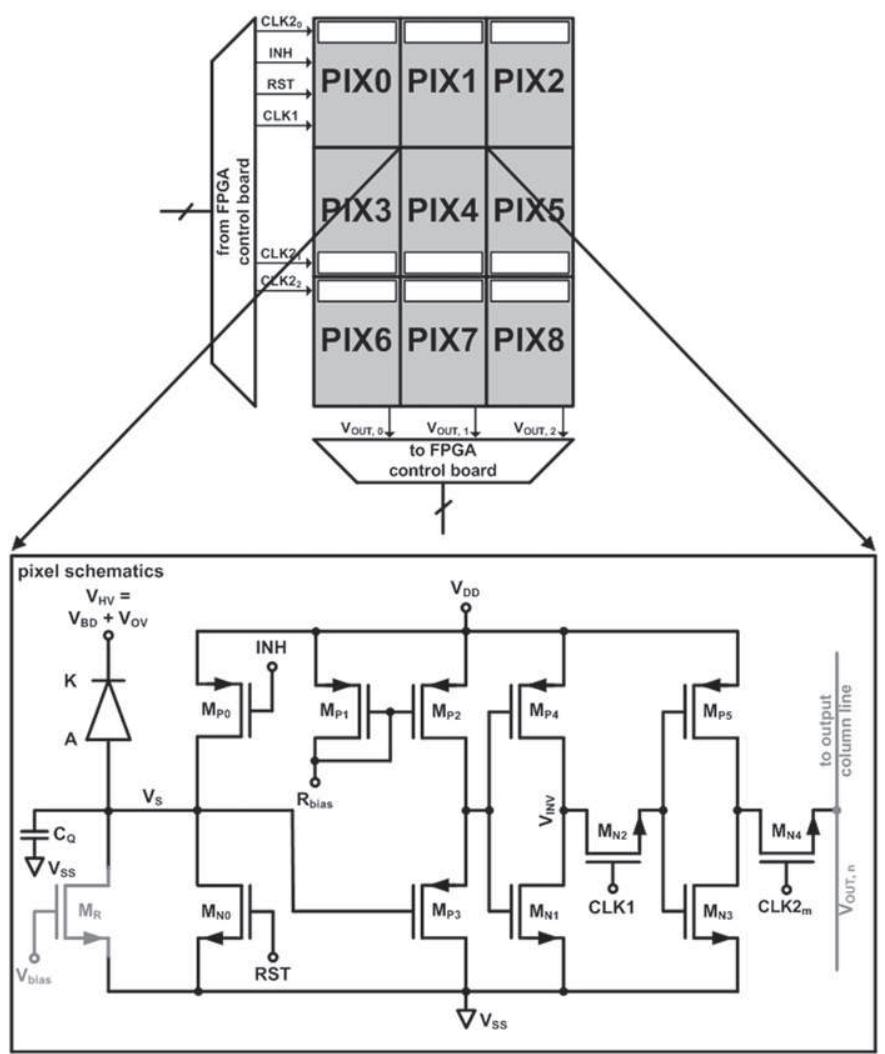

Fig. 1. Block diagram of the proposed $3 \times 3$ SPAD array together with the schematics of the proposed digital pixel. given the extreme dependence of the DCR on the cleanness of the fabrication process, an excessively irregular DCR distribution may appear amongst the pixels of bidimensional arrays of SPADs [9]. Punctual defects in the crystal lattice, such as clusters of impurities or dislocations, may also lead to defective pixels with noise levels well above the acceptable threshold. These pixels, called dead pixels or hot pixels, represent a serious problem for SPAD detectors as they reduce the yield.

Solutions commonly adopted to reduce the noise in SPAD detectors regard dedicated technologies with lower doping profiles [10], cooling methods either with Peltier elements [11] or air cooling [12], and advanced front-end circuits that minimize the avalanche charge flow [13]. Dead pixels are simply switched off by means of digital processing [3]. However, none of the presented techniques is completely satisfactory given the high fabrication costs of dedicated technologies, the reduced applicability of cooling methods, the limited efficiency of advanced front-end circuits or the severe loss of sensitive area induced by switching off dead pixels.

Apart from that, in those applications where the signal arrival time is known, the sensor can also be operated in the gated acquisition mode. In contrast with the free-running mode of operation, where the sensor is always reverse biased above $V_{\mathrm{BD}}$ at a fixed voltage, in the gated acquisition the reverse bias voltage swings from over to under $V_{\mathrm{BD}}$ to periodically enable and disable the photodiode. The sensor is then kept active only for short periods of time that can be synchronized with the expected signal arrival. Consequently, the probability to detect dark counts interfering with signal triggered counts (known as dark count probability or DCP) is linearly reduced with the width of the active period of the sensor without missing any photon counts. In addition, long enough non-active periods, longer than the lifetime of the trapping levels, allow to completely release trapped charges and therefore to eliminate the afterpulsing probability. In this article, we propose the gated mode of acquisition to synchronize sensor operation with the expected signal arrival, to reduce the DCP and also to get rid of afterpulses in CMOS SPAD bidimensional arrays. In addition, the reduction of the DCP also allows to increase the detector performance.

\section{Array design and operation}

The block diagram of the proposed SPAD detector together with the schematics of the pixels is shown in Fig. 1. The detector consists of an array of $3 \times 3$ pixels. It was designed and fabricated with the standard HV-AMS $0.35 \mu \mathrm{m}$ CMOS technology (h35b4). Each pixel combines a rectangular SPAD with a sensitive area of $20 \mu \mathrm{mx} 100 \mu \mathrm{m}$ and the proper readout electronics. The photodiode is implemented by means of a $\mathrm{p}^{+} /$deep $\mathrm{n}$-tub junction, which

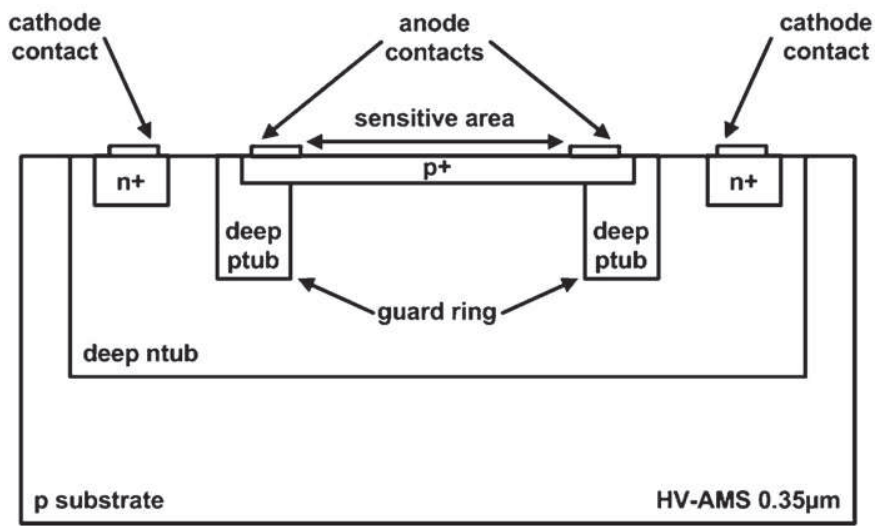

Fig. 2. Cross section of the SPAD fabricated with the standard HV-AMS $0.35 \mu \mathrm{m}$ CMOS technology. 
is surrounded by a p-tub implantation set to prevent premature edge breakdown (see Fig. 2 for SPAD cross section). Additionally, the corners of the sensor are rounded to avoid electric field peaks at the junction corners. The pixels of rows 0 and 1 share the deep n-tub layer, as well as the pixels of row 2 share another one, to obtain a reduced pitch $(22.9 \mu \mathrm{m})$ and an improved fill factor $(90.2 \%)$. The breakdown voltage of the junction is measured to be at $18.9 \mathrm{~V}$, while a $V_{\mathrm{DD}}$ of $3.3 \mathrm{~V}$ powers the rest of the pixel. Reverse bias voltages of $V_{\mathrm{BD}}+V_{\mathrm{OV}}$ are applied to the sensor cathode to operate the Geiger mode. The readout is performed at the anode or sensing node $\left(V_{S}\right)$. The transistor $M_{R}$ was included to study the response of the detector for different recharge times, achieved through an externally adjustable $V_{\text {bias }}$, but it is not used in the gated mode of operation. It could be removed to minimize the area occupation as well as the charge flowing during an avalanche.

The benefits of the gated operation with commercially available germanium [14] and InGaAs/InP [15] APDs for the detection of 1-1.3 $\mu \mathrm{m}$ wavelengths have already been discussed in the literature. In these cases, short gate pulses are achieved with voltage generators. Other possibilities to apply the gating pulse are based on AC coupling [16] or high frequency sinousoidal voltages [17], but whereas the former imposes a limitation on the repetition rate due to the coupling capacitor recharge time constant, the latter keeps variable the reverse bias of the sensor during gating periods. Recently, CMOS SPAD detectors with monolithically integrated electronics to operate the gated mode were also reported for lifetime measurements $[18,19]$. However, a large number of transistors per pixel is needed. We previously claimed the efficiency of the gated operation in SPAD detectors based on single pixels [20].

In the SPAD bidimensional array proposed in this work, the gated operation is controlled by means of two external signals implemented through MOS transistors. When turned on by the RST signal, the transistor $M_{\mathrm{N} 0}$ quickly recharges the sensor ( $<1 \mathrm{~ns}$ ) by increasing its bias over $V_{\mathrm{BD}}$ and the gated 'on' period is started. However, given that avalanches can still happen while the RST transistor is on, the RST pulse has to be as short as possible in order to minimize the presence of low resistive paths from $V_{\mathrm{S}}$ to ground $\left(V_{\mathrm{SS}}\right)$ and ensure a robust quenching. On the contrary, when the INH signal switches on transistor $M_{\mathrm{PO}}$, the polarization of the sensor is reduced under $V_{\mathrm{BD}}$, defining this way the gated 'off' period. When an avalanche is triggered, the self-sustained current that flows through the junction charges the parasitic capacitance $\left(C_{Q}\right)$ of $V_{\mathrm{S}}$ in picoseconds until its voltage raises up to $V_{\mathrm{OV}}$. At this point, the polarization of the sensor drops down to $V_{\mathrm{BD}}$ and the avalanche is quenched. The node $V_{S}$ is connected to the readout electronics, which is based on a level-shifter $\left(M_{\mathrm{P} 1}, M_{\mathrm{P} 2}\right.$ and $\left.M_{\mathrm{P} 3}\right)$ and a CMOS inverter $\left(M_{\mathrm{P} 4}\right.$ and $\left.M_{\mathrm{N} 1}\right)$. A low $V_{\mathrm{OV}}$ is desired to reduce the DCR. However, it is not allowed in this technology given that the threshold voltage of nMOS transistors is set at $0.5 \mathrm{~V}$. In order to overcome this drawback, the level-shifter externally biased was included to rise the avalanche voltage $\left(V_{\mathrm{OV}}\right)$ over the threshold voltage of the following CMOS inverter, which is set at $V_{\mathrm{DD}} / 2$. The output of the inverter $\left(V_{\mathrm{INV}}\right)$ is feed in a dynamic latch $\left(M_{\mathrm{N} 2}, M_{\mathrm{P} 5}\right.$ and $\left.M_{\mathrm{N} 3}\right)$ which has been included to make possible in-pixel storage. The dynamic latch is synchronized with the active periods of the sensor by means of the external signal CLK1. This signal switches on the gate $M_{\mathrm{N} 2}$ at the beginning of the gated 'on' period and switches it off a few nanoseconds before the gated 'off' period is started to avoid storing a false ' 1 '. The interval of time in which the dynamic latch is sampling the inverter output is called period of observation $\left(t_{\mathrm{obs}}\right)$. The value of $V_{\mathrm{S}}$ at the end of $t_{\mathrm{obs}}$ is stored during the gated 'off' period. The gating periods are globally applied to all the pixels simultaneously. The waveforms to control the SPAD in the gated operation are depicted in Fig. 3.

The three rows of the SPAD array are read sequentially during the gated 'off' periods of the sensor. Thereby, the three columns of

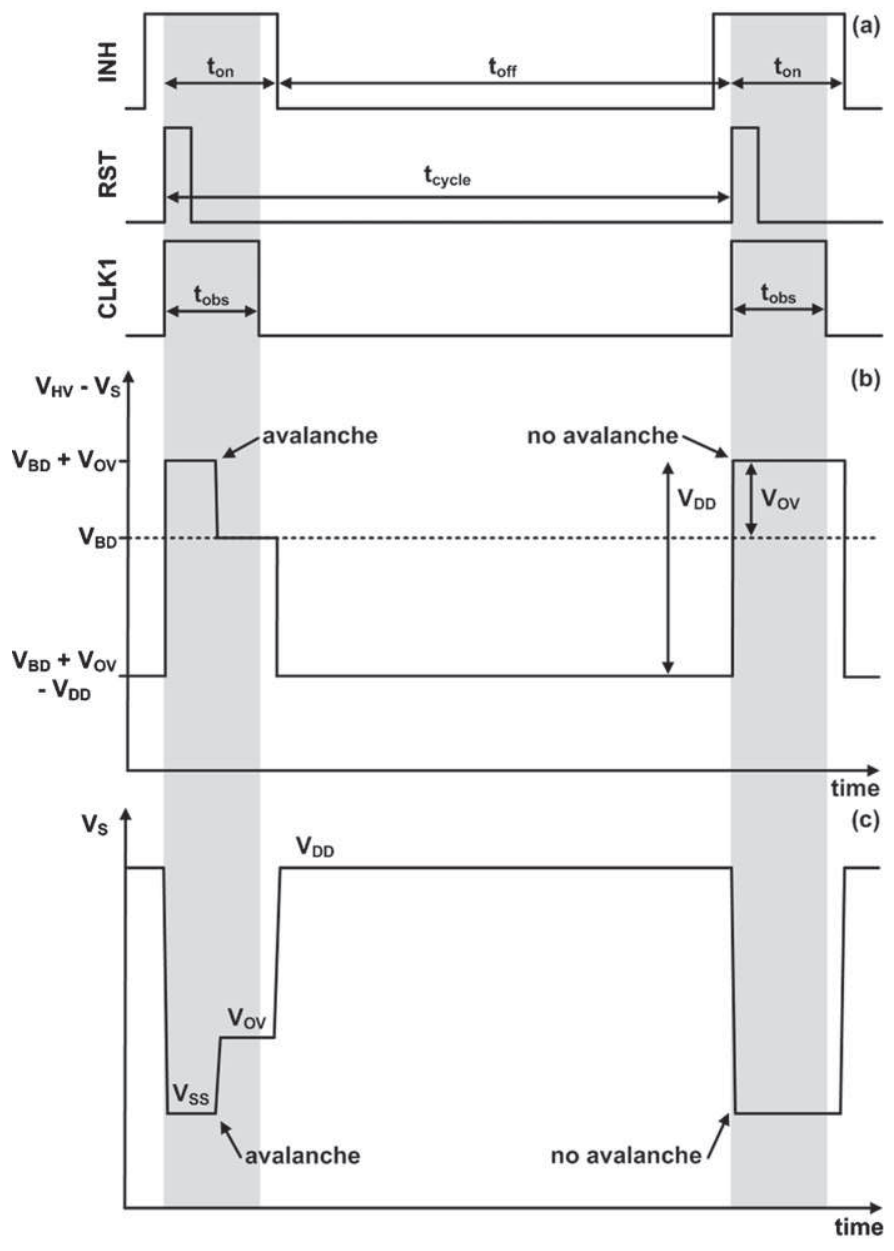

Fig. 3. Waveforms for the gated operation (a), SPAD bias (b) and voltage of the sensing node $(c)$.

each row are read in parallel, requiring only three output pads. To allow external selection of the row $m$ to be read, a simple address circuit based on a pass gate $\left(M_{\mathrm{N} 4}\right)$ activated by the $C L K 22_{\mathrm{m}}$ signal has been placed between the dynamic latch and the output column line. The pass gate contacts within one row are connected together and therefore switched on simultaneously. When triggered by $C L K 2_{m}$ (i.e., $C L K 2_{m}=' 1$ '), each one of the selected pass gates feeds its corresponding output column line, which is directly connected to the output pad. Neither multiplexers nor selection decoders are needed. Despite the small number of pixels, the presented array is a demonstrator of a larger bidimensional camera.

\section{Results and discussion}

A micrograph of the prototype fabricated with the standard HV-AMS $0.35 \mu \mathrm{m}$ CMOS technology can be seen in Fig. 4. To demonstrate the efficiency of the proposed methods to reduce the noise in SPAD detectors, the response of the pixel in darkness and also to light was tested at room temperature. The chip was mounted on a printed circuit board and powered with an Agilent E3631A voltage source. An ALTERA Stratix II FPGA-based control board was used to generate the fast logic control signals (RST, INH, CLK1 and CLK2 $\mathrm{m}$ ) and also to count off-chip the number of pulses generated by the sensor. The optical response of the pixel to a variable intensity of a $880 \mathrm{~nm}$ light was studied by placing an infrared LED above the sensor. The light emitter was powered using an HP 3245A universal source and the current flowing through it was measured by means of an HP 3458A multimeter. The chip, together with the FPGA and 


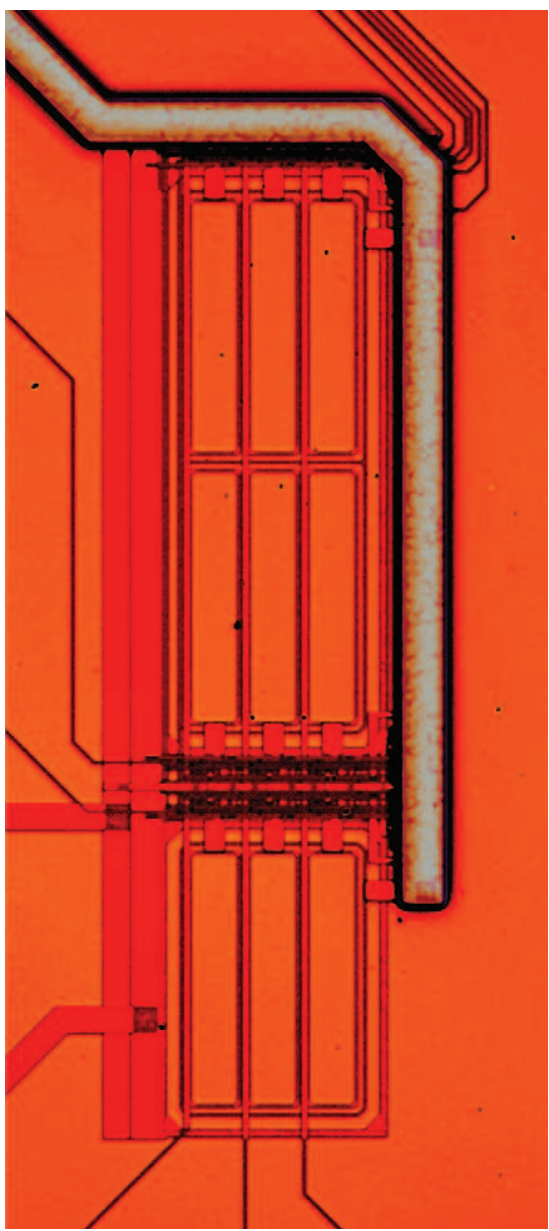

Fig. 4. Micrograph of the fabricated $3 \times 3$ SPAD array.

the infrared LED, was placed inside a metallic box to protect the circuit from electromagnetic interferences and uncontrolled light sources. The measurements were done with an adjustable integration time that depends on the period of observation of the sensor and also on the number of times that the observation is repeated $\left(n_{\text {rep }}\right)$. Different $t_{\text {obs }}$ that range from 10 ns to 1280 ns were analyzed for different $V_{\mathrm{OV}}$.

Firstly, the afterpulsing probability was tested by leaving different gated 'off' periods for a fixed $t_{\mathrm{obs}}$ of $10 \mathrm{~ns}$. It was observed that

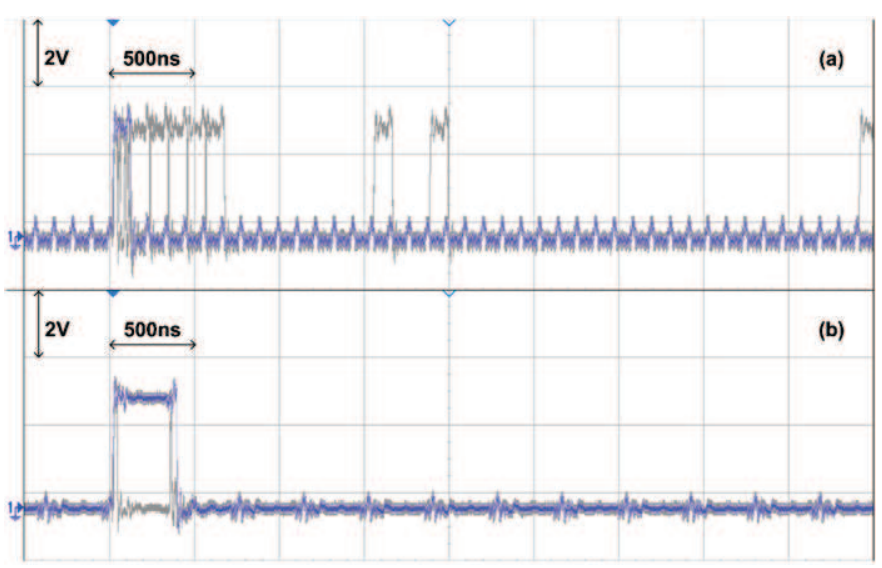

Fig. 5. Presence of afterpulses with a $t_{\text {off }}$ of $80 \mathrm{~ns}$ (a) and elimination of afterpulses with a $t_{\text {off }}$ of $300 \mathrm{~ns}$ (b) observed at the pixel output. Measured with the infinite permanence option of the oscilloscope.

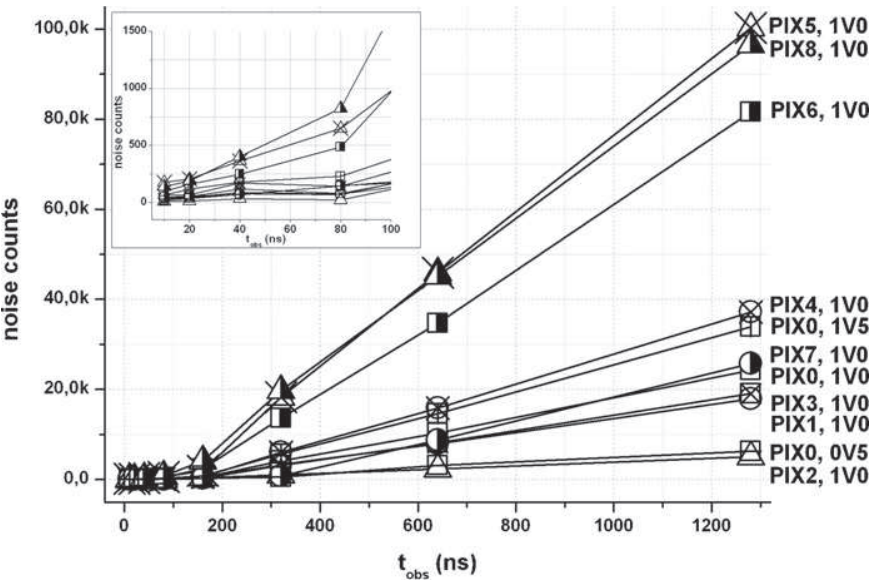

Fig. 6. Noise counts of the different pixels of the array for different $t_{\mathrm{obs}}$ and $V_{\mathrm{OV}}$.

gated 'off' periods of $300 \mathrm{~ns}$ are enough to eliminate afterpulses at a $V_{\mathrm{OV}}$ of $1.0 \mathrm{~V}$ (Fig. 5), which shows that the lifetime of the trapping levels is shorter than this time. Secondly, the dark counts of the detector were measured for different $t_{\mathrm{obs}}$ with a fixed $t_{\mathrm{off}}$ of $300 \mathrm{~ns}$ and different $V_{\mathrm{OV}}$ of $0.5 \mathrm{~V}, 1.0 \mathrm{~V}$ and $1.5 \mathrm{~V}$. To obtain a statistical population, $400 \mathrm{k}$ repetitions were performed. As expected, dark counts are reduced for a lower $V_{\mathrm{OV}}$ (Fig. 6). Moreover, dark counts are lessened linearly with shorter $t_{\mathrm{obs}}$, as it can be inferred from dark counts $=\mathrm{DCR} \times t_{\mathrm{obs}}$. Taking for example a DCR of $50 \mathrm{kHz}$, with a $t_{\mathrm{obs}}$ of $10 \mathrm{~ns}$ only one dark count will be seen each 2000 repetitions of the measurement. For a $t_{\mathrm{obs}}$ of $20 \mathrm{~ns}$, this rate increases up to 1000 repetitions, and so on for longer $t_{\text {obs }}$. Consequently, in those applications where the signal to be detected is present only in a well defined interval after a triggering signal, the gated operation with discrete $t_{\mathrm{obs}}$ in the nanosecond range allows to dramatically reduce the probability to detect dark counts without missing any photon count. It can also be observed that noise discrepancies amongst the pixels of the array are large (more than a factor 20 between the most and the less noisy ones), as it usually happens in SPAD arrays.

The response of the detector to light was tested for two different $t_{\mathrm{obs}}$ of $10 \mathrm{~ns}$ and $1280 \mathrm{~ns}$ for a fixed $V_{\mathrm{OV}}$ of $1.0 \mathrm{~V}$ with a counter of a maximum capacity of 8000 counts. For each $t_{\mathrm{obs}}$, the detector was illuminated with different light intensities and its response was observed for 8000 times. The measurement was repeated 10 times to obtain enough statistics. The mean values of the experimental

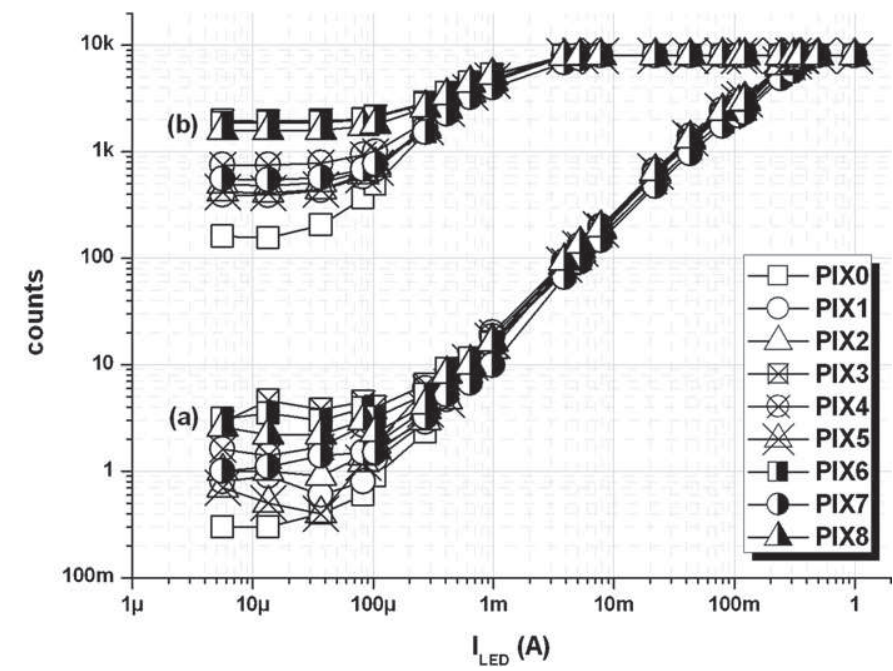

Fig. 7. Response of the SPAD detector to a light intensity of $880 \mathrm{~nm}$ at $1.0 \mathrm{~V}$ of $V_{\mathrm{OV}}$ for different $t_{\mathrm{obs}}$ of $10 \mathrm{~ns}$ (a) and $1280 \mathrm{~ns}$ (b). 


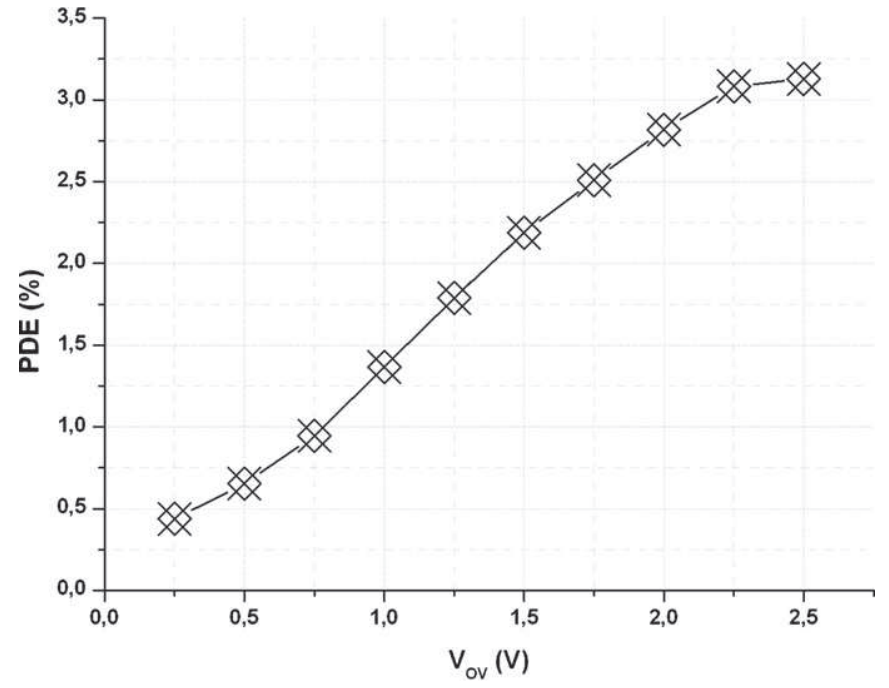

Fig. 8. Photon detection efficiency measured at different $V_{\mathrm{OV}}$ for a light intensity of $880 \mathrm{~nm}$.

data are plotted in Fig. 7, where the number of counts has been depicted as a function of the LED intensity. At low intensities, the detected counts are only noise counts and no signal counts are appreciated. The threshold intensity $\left(I_{\mathrm{th}}\right)$ corresponds to the minimum intensity from which signal counts above the noise floor are obtained. Several light intensities were tested until the generated counts caused counter saturation. The intensity that causes counter saturation corresponds to the saturation intensity $\left(I_{\mathrm{sat}}\right)$. As shown in Fig. 7, the measurements with shorter $t_{\text {obs }}$ generate lower noise floors. Despite this variation in the noise floor, $I_{\text {th }}$ is independent of the width of the $t_{\mathrm{obs}}$ time and measured to be $13 \mu \mathrm{A}$. However, shorter $t_{\mathrm{obs}}$ generate higher $I_{\mathrm{sat}}$. For the $1280 \mathrm{~ns}$ case, $I_{\mathrm{sat}}$ is measured to be $7.7 \mathrm{~mA}$, whereas for the $10 \mathrm{~ns}$ case $I_{\mathrm{sat}}$ is $0.35 \mathrm{~A}$. Due to a reduced noise floor because of the shorter $t_{\mathrm{obs}}$, the range of intensities in which the sensor is sensitive to light is extended at the high end.

The maximum detectable signal is reached at $I_{\text {sat }}$, whereas the minimum is reached at $I_{\text {th }}$. Hence, the dynamic range (DR) of a gated detector, which is active only for short periods of time that are synchronized with the expected signal arrival, can be obtained by dividing the maximum detectable signal received after the trigger event by the threshold intensity. It can be expressed as DR $=\log _{2}\left(I_{\mathrm{sat}} / I_{\mathrm{th}}\right)$. The DR amongst the different pixels of the detector is of 10 bits for the 1280 ns case. In contrast, this parameter is increased up to 15 bits for the $10 \mathrm{~ns}$ case. The DR is not regarded as the response of the detector to light only, as identical total measurement times give the same result despite the period of observation used. Provided that the signal arrival is known, as it happens in applications with triggering signals, the measurements are taken during a few nanoseconds after the trigger events only. Measurements taken with short $t_{\mathrm{obs}}$ yield an extension of the DR, and consequently a better resolution of the pixel, than those ones taken with longer $t_{\mathrm{obs}}$.

In the last place, the sensitivity of the detector as a function of $V_{\mathrm{OV}}$ was also measured for a fixed wavelength of $880 \mathrm{~nm}$. Fig. 8 shows the mean value of the photon detection efficiency (PDE) of the different pixels of the detector as a function of $V_{\mathrm{OV}}$. The presented values are in good agreement with the literature [18]. As expected, the PDE increases with $V_{\mathrm{OV}}$ until saturation. However, low $V_{\mathrm{OV}}$ of $1.0 \mathrm{~V}$ (PDE of 1.4\%) are good enough to allow photon detection.

\section{Conclusion}

A bidimensional array based on SPADs operated in the gated mode for triggered systems has been designed and fabricated with the $0.35 \mu \mathrm{m}$ HV-AMS standard technology. It has been demonstrated that the proposed mode of operation with short gated 'on' periods allows to eliminate the afterpulsing probability and to minimize the triggering of dark counts. A readout circuit to operate the sensor with low reverse bias overvoltages allows to reduce the dark count rate. Using periods of observation of $10 \mathrm{~ns}$, the detector performance is highly improved, presenting an extended dynamic range of 15 bits $\left(V_{\mathrm{OV}}=1.0 \mathrm{~V}\right)$. A good enough photon detection efficiency of $1.4 \%$ has been measured with a reverse bias overvoltage of $1.0 \mathrm{~V}$. It is concluded that the gated operation allows to reduce noise figures while the sensitivity of the sensor is good enough.

\section{Acknowledgments}

This work has been partially supported by the National Program for Particle Physics through the projects "Desarrollo de nuevas tecnologías en aceleradores y detectores para los futuros colisionadores de Física de Partículas", coded FPA2008-05979-C04-02, and "Desarrollo de nuevos detectores para los Futuros colisionadores en Física de Partículas", coded FPA2010-21549-C04-01.

\section{References}

[1] K.D. Stefanov, CCD-based vertex detector for the future linear collider, Nucl. Instrum. Methods Phys. Res. Sect. A 549 (2005) 93-98.

[2] G. Deptuch, G. Claus, C. Colledani, M. Deveaux, A. Gay, W. Dulinski, W. Gornushkin, C. Hu-Guo, M. Winter, Development of monolithic active pixel sensors for charged particle tracking, Nucl. Instrum. Methods Phys. Res. Sect. A 511 (2003) 240-249.

[3] T. Frach, G. Prescher, C. Degenhardt, R. Gruyter, A. Schmitz, R. Ballizany, The digital photomultiplier-principle of operation and intrinsic detector performance, IEEE Nucl. Sci. Symp. Conf. Record (2009) 1959-1965.

[4] Y. Banda, P. Coulter, D. Cussans, C. Damerell, E. Devetak, J. Fopma, B. Foster, R. Frost, R. Gao, J. Goldstein, T. Greenshaw, K. Harder, B. Hawes, S. Hillert, B. Jeffery, J.J. John, N. Kundu, Y. Li, P. Murray, A. Nomerotski, C. Perry, K. Stefanov, S. Thomas, J. Velthuis, T. Wolliscroft, S. Worm, J. Yow, Z. Zhang, Design and performance of improved column parallel CCD, CPC2, Nucl. Instrum. Methods Phys. Res. Sect. A 621 (2010) 192-204.

[5] J.J. Velthuis, R. Kohrs, M. Mathes, A. Raspereza, L. Reuen, L. Andricek, M. Koch, Z. Dolezal, P. Fischer, A. Frey, F. Giesen, P. Kodys, C. Kreidl, H. Krüger, P. Lodomez, G. Lutz, H.G. Moser, R.H. Richter, C. Sandow, D. Scheirich, E. von Törne, M. Trimpl, Q. Wei, N. Wermes, DEPFET, a monolithic active pixel sensor for the ILC, Nucl. Instrum. Methods Phys. Res. Sect. A 579 (2007) 685-689.

[6] F. Zappa, S. Tisa, A. Tosi, S. Cova, Principles and features of single-photon avalanche diode arrays, Sens. Actuators. A 140 (2007) 103-112.

[7] A. Rochas, M. Gani, B. Furrer, P.A. Besse, R.S. Popovic, G. Ribordy, N. Gisin, Single photon detector fabricated in a complementary metal-oxide-semiconductor high-voltage-technology, Rev. Sci. Instrum. 74 (2003) 3263-3270.

[8] E. Vilella, A. Arbat, A. Comerma, J. Trenado, O. Alonso, D. Gascon, A. Vilà, L. Garrido, A. Diéguez, Readout electronics for low dark count pixel detectors based on Geiger mode avalanche photodiodes fabricated in conventional CMOS technologies for future linear colliders, Nucl. Instrum. Methods Phys. Res. Sect. A 650 (2011) 120-124.

[9] A. Rochas, M. Gösch, A. Serov, P.A. Besse, R.S. Popovic, T. Lasser, R. Rigler, First fully integrated 2-D array of single-photon detectors in standard CMOS technology, IEEE Photon. Technol. Lett. 15 (2003) 963-965.

[10] M. Gersbach, J. Richardson, E. Mazaleyrat, S. Hardillier, C. Niclass, R. Henderson, L. Grant, E. Charbon, A low-noise single-photon detector implemented in a $130 \mathrm{~nm}$ CMOS imaging process, Solid-State Electron. 53 (2009) 803-808.

[11] T. Yoshida, T. Sora, A prototype avalanche photodiode array for scintillatingfiber tracking detectors, Nucl. Instrum. Methods Phys. Res. Sect. A 534 (2004) 397-402.

[12] S. McCallum, P. Clowes, A. Welch, A four-layer attenuation compensated PET detector based on APD arrays without discrete crystal elements, Phys. Med. Biol. 50 (2005) 4187-4207.

[13] S. Tisa, F. Guerrieri, F. Zappa, Variable-load quenching circuit for single-photon avalanche diodes, Opt. Express 16 (2008) 2232-2244. 
[14] G.S. Buller, S.J. Fancey, J.S. Massa, A.C. Walker, S. Cova, A. Lacaita, Time-resolved photoluminescence measurements of InGaAs/InP multiple-quantum-wel structures at $1.3 \mathrm{~m}$ wavelengths by use of germanium single-photon avalanche photodiodes, Appl. Opt. 35 (1996) 916-921.

[15] A. Lacaita, F. Zappa, S. Cova, P. Lovati, Single-photon detection beyond $1 \mathrm{~m}$ : performance of commercially available InGaAs/InP detectors, Appl. Opt. 35 (1996) 2986-2996.

[16] O. Thomas, Z.L. Yuan, J.F. Dynes, A.W. Sharpe, A.J. Shields, Efficient photon number detection with silicon avalanche photodiodes, Appl. Phys. Lett. 97 (2010) 031102.

[17] N. Namekata, S. Sasamori, S. Inoue, $800 \mathrm{MHz}$ single-photon detection at $1550 \mathrm{~nm}$ using an InGaAs/InP avalanche photodiode operated with a sine wave gating, Opt. Express 14 (2006) 10043-10049.

18] D. Stoppa, D. Mosconi, L Pancheri, L Gonzo, Single-photon avalanche diode CMOS sensor for time-resolved fluorescence measurements, IEEE Sens. J. 9 (2009) 1084-1090.

[19] Y. Maruyama, E. Charbon, An all-digital, time-gated $128 \times 128$ spad array for on-chip, filter-less fluorescence detection, in: 16th International Conference on Solid-State Sensors, Actuators and Microsystems, 2011, pp. 1180-1183.

[20] E. Vilella, A. Comerma, O. Alonso, A. Diéguez, Low-noise pixel detectors based on gated Geiger mode avalanche photodiodes, Electron. Lett. 47 (2011) 397-398.

\section{Biographies}

E. Vilella received the BSc degree in Electronic Engineering from the University of Barcelona (UB), Spain, in 2009 and a MSc degree also in Electronic Engineering from the same University in 2010. She is currently working toward the PhD in Engineering and Applied Technologies at UB. Her research interests include the development of low voltage particle tracking pixel detectors based on avalanche photodiodes for future linear colliders such as the International Linear Collider (ILC) and the Compact LInear Collider (CLIC).

A. Diéguez is currently Associate Professor in the University of Barcelona, Spain. He obtained his Physics degree in 1993 by the University of Barcelona. In 1999 he obtained his PhD in Physics from the same University. His research activities during these years have been developed mainly in the field of basic semiconductor research and microelectronic circuit design. He has participated actively in 6 European projects and has more than 40 papers related to the activities commented. From 1993 to 1999 he developed his activity in the field of semiconductor gas sensors. Since 1998 his research activity is devoted to the design of Application Specific Integrated Circuits. The main areas of interest are mixed-signal design of ICs and low-power VLSI design. Specific research topics are microrobotics, low-power smart sensor systems, radiation tolerant electronics and vision systems. 